\title{
Benefits of Bilateral Electrical Stimulation with the Nucleus Cochlear Implant in Adults: 6-Month Postoperative Results
}

\author{
*Roland Laszig, *Antje Aschendorff, *Matthias Stecker, †Joachim Müller-Deile, \\ $\dagger$ Steffen Maune, $\ddagger$ Norbert Dillier, $\ddagger$ Benno Weber, §Matthias Hey, \\ $\S$ Klaus Begall, "Thomas Lenarz, "Rolf-D. Battmer, "Melanie Böhm, \\ ITThomas Steffens, IJJuergen Strutz, \#Thomas Linder, **Rudolf Probst, \\ $* *$ John Allum, $\dagger \dagger$ Martin Westhofen, and $\dagger \dagger$ Wolfgang Doering \\ *HNO Klinik Universität Freiburg, †HNO Klinik Universitätsklinikum Kiel, §HNO Klinik St. \\ Salvator-Krankenhaus Halberstadt, "HNO Klinik Medizinische Hochschule Hannover, 9IHNO Klinik \\ Universitätsklinikum Regensburg, **HNO Universitätsklinik Basel, and $\dagger \dagger H N O$ Klinik \\ Universitätsklinikum Aachen, Germany; and $\ddagger$ HNO Klinik Universitätsspital Zürich and \#HNO Klinik \\ Kantonspital Luzern, Switzerland
}

\begin{abstract}
Objective: To evaluate the benefits of bilateral electrical stimulation for hearing-impaired adult subjects using the Nucleus 24 cochlear implant in a multicenter study, and to compare and quantify performance on speech perception measures in quiet and in noise and localization ability for unilateral and bilateral cochlear implant use.

Design: Repeated single subject measures were carried out for each subject, with each subject serving as their own control. Assessment of unilateral and bilateral listening conditions for performance on tests of speech comprehension and sound localization were performed. Speech comprehension measures were performed in quiet at 0 degree azimuth and in the presence of background noise simultaneously presented from the same speaker and spatially separated by 90 degrees, at $\mathrm{S}+45^{\circ} \mathrm{N} 45^{\circ}$ and at $\mathrm{S}-45^{\circ} \mathrm{N}+45^{\circ}$. Test materials included Freiburger monosyllabic words, Oldenburger sentences, and the Hochmair-Schulz-Moser sentences. Tests of localization were performed in the horizontal plane with 12 speaker locations 30 degrees apart using a shortened sentence stimulus from the Hochmair-Schulz-Moser sentences at two possible presentation levels of 55 and $70 \mathrm{~dB}$ sound pressure level for assessment of directionality. The binaural advantage provided by bilateral stimulation was calculated with respect to each ear separately, classified as either the better or poorer performing ear for each speech material in quiet and in noise test conditions. For localization of sound, the binaural advantage was compared with left and right ears separately. Paired comparisons for performance data in all conditions were carried out by considering measurements for each subject in different condi-
\end{abstract}

Address correspondence and reprint requests to Prof. Dr. Dr. h.c. Roland Laszig, M.D., Universitätsklinik für HNO und Poliklinik, Elsaesser Strasse 2N, Freiburg 71190, Germany; E-mail: laszig@hno1. ukl.uni-freiburg.de tions as paired observations and applying the Student's $t$ test to determine the statistical difference between the data sets.

Setting: Tertiary referral centers with a cochlear implant program. Patients: Thirty-seven profoundly hearing-impaired adults were enrolled in the study, 22 simultaneously and 15 sequentially bilaterally implanted. All patients received the Nucleus 24 cochlear implant and used the Nucleus SPrint or ESPrit 3G speech processor, with the vast majority using the ACE speech coding strategy.

Results: For spatially separated speech in noise conditions, an interaural performance advantage for the ear closest to the speech source (i.e., with a superior signal to noise ratio) compared with that for the ear closest to the noise source (i.e., with an inferior signal to noise ratio) is consistently demonstrated regardless of whether it is the better or poorer performing ear closest to the speech signal. This is referred to as a significant binaural head-shadow benefit, resulting in a mean improvement between $-10 \mathrm{~dB}$ and $-11.4 \mathrm{~dB}$ in the critical signal to noise ratio required for $50 \%$ speech comprehension for the Oldenburger sentences and a mean improvement in the maximum score of $42 \%$ to $55 \%$ for the ear closest to the speech signal over the ear farthest away for the Hochmair-Schulz-Moser sentences. Bilateral stimulation is always observed to provide a performance advantage over the unilateral listening condition for either ear when ipsilateral to the noise source. In addition, as demonstrated by approximately half the subjects tested in noise with the Hochmair-Schulz-Moser sentences, a performance advantage of bilateral stimulation may be observed over the better ear alone when positioned ipsilateral to the speech signal, which is referred to as a binaural squelch effect. On average, for the group, this resulted in a statistically significant improvement in speech comprehension scores of $8 \%$ in the bilateral listening condition compared with the scores for the better ear alone. Through assessment of comprehension of coincidental speech in noise and speech in quiet, a significant 
benefit of binaural redundancy was noted for the group for Oldenburger sentence scores in noise and in quiet compared with unilateral scores for either ear and for the Freiburger monosyllabic words in quiet in comparison with the better ear alone scores. Binaural stimulation also led to a significant improvement in localization ability over either monaural condition, with the root mean square degrees of error reduced by 38 degrees compared with that observed for unilateral stimulation. Conclusion: Similar to what has been observed for bilateral acoustic stimulation in the past, bilateral electrical stimulation provides the foundation for the potential advantages of the head-shadow effect, providing a binaural head-shadow benefit and binaural auditory processing such as binaural redundancy and binaural squelch effects, all of which combine to lead to improved speech comprehension over unilateral listening conditions. The combination of improved speech comprehension and improved localization ability made available through bilateral electrical stimulation provides the necessary foundation to further assist the hearing-impaired listener to better cope with communication in the everyday listening situation both in noise and in quiet. Key Words: Bilateral cochlear implantsBinaural electrical stimulation-Nucleus CI24M.

Otol Neurotol 25:958-968, 2004.
Research has established over the years the advantages of binaural hearing for normal listeners, and the subsequent benefits of bilateral acoustic and bone conduction amplification for the hearing-impaired are measurable both objectively and subjectively. The benefit of listening with both ears is particularly evident for speech comprehension performance measures carried out for spatially separated speech in noise test conditions and at a relatively poor signal-to-noise ratio for the individual (1-4). More recently, efforts have also been directed toward demonstrating the binaural advantage for localization of sound for both normal and hearing-impaired listeners that may ultimately assist the individual's sense of well-being in their environment $(4,5)$.

Despite the clear need for and proven advantages of binaural hearing for speech comprehension, particularly for the hearing-impaired individual in the everyday listening environment that is neither optimal nor static in its configuration, relatively little research has been specifically directed toward assessing the benefits of bilateral stimulation for cochlear implantees (6-14). As Tyler et al. (10), van Hoesel et al. (11), Schön et al. (9), and Mueller et al. (8) have shown in single-site studies involving small subject groups $(\mathrm{n}=1-9)$, bilateral stimulation provides measurable advantages for speech comprehension in a variety of situations, particularly in spatially separate speech in noise conditions. In addition, single-site studies have demonstrated the benefit of bilateral stimulation for the lateralization and localization of sound relative to monaural stimulation in the sound field for cochlear implant patients (10-13). The only multicenter study reported to date, by Stark et al. (14), echoes bilateral benefits for speech comprehension in noise and in quiet test conditions similar to the previous single-site studies. Across the variety of test setups used, the strongest and most robust effect demonstrated for spatially separated speech in noise tests is the headshadow effect, resulting in the subject's attention to the information available from the ear with the most favorable signal-to-noise ratio $(2,8,12)$. Although the headshadow effect is not a consequence of binaural sound processing, it is a consequence of wearing two devices, which permits the subject to take advantage of the presence of two different signal-to-noise ratios, subsequently leading to improved speech comprehension, and can thus be referred to as a bilateral head-shadow benefit. For the binaurally implanted patient, as for the normal listener or the hearing aid user, the head-shadow effect primarily influences the intensity level of the high-frequency components of sound on the shadowed side of the head and gives the patient the flexibility to attend to either implanted ear pending the changing characteristics of the sound environment at any given time.

True auditory processing effects of listening with two ears, binaural squelch and binaural redundancy, appear to be much harder to demonstrate for cochlear implant subjects and, when present, are much weaker and less robust for the subject groups $(8,13)$. Binaural squelch results from the brain's ability to take advantage of varying auditory inputs to both ears in situations where the speech and noise are spatially separated, thus creating differing timing and intensity cues for each ear (2). Subsequently, the brain is able to better sort out the speech signal from the noise signal in trying to comprehend speech. The fact that this is a measurable advantage for normal listeners and either not measurable or very small for bilateral cochlear implant subject groups studied to date may suggest additional influences. Perhaps the output signal of the speech processor, which may preserve the timing and intensity information to varying degrees, in combination with altered ability for the hearingimpaired individual to use such cues once received is involved $(8,9)$. In addition, strong influences of interaural asymmetry may reduce the effect, as the better ear may dominate and the subject may then attend to the better ear only $(2,12)$.

In contrast to both head-shadow and binaural squelch effect, binaural summation, resulting from the effect of binaural loudness summation and binaural redundancy, occurs when the same auditory input arrives at each ear with the same auditory characteristics (2). Subsequently, the brain is able to make use of the overlapping information to comprehend speech better. Although binaural loudness is a pure summation of the intensity of the signal arriving at each ear simultaneously, binaural redundancy represents binaural processing of the two signals. To remove the influence of binaural loudness summation on performance as far as possible, loudness balancing of unilaterally and bilaterally used processor programs (map) is required per individual. Most studies 
to date report on a small (1-2 dB) measurable binaural summation for only a portion of the bilateral subjects studied, taking into account loudness balancing during the programming for each listening condition (12). A relatively larger effect was noted for the study group when the loudness was not balanced between the individual processor maps $(8,9)$. Similar to binaural squelch, the interpretation of binaural redundancy will be affected by significant interaural asymmetries for the test measures (2).

The ability to localize sound, which is reliant on the ability to use timing and intensity cue differences between the auditory signals arriving at each ear, is clearly a much easier exercise when binaurally stimulated than in the monaural condition for most subjects, given that the ears are fairly symmetric $(2,5,10-12)$. Studies of localization ability for bilateral cochlear implant (CI) subjects report differing test setups, involving various locations, speaker numbers, and stimuli. The studies suggest a clear binaural advantage for the majority of individuals and most certainly for the subject groups. The root mean square (RMS) error in degrees for the overall responses in relation to the stimuli speaker locations for each listening condition varies between the studies, most likely as a consequence of the largely varying test methods and setups, but the relative binaural advantage was always significant.

To date, over 50,000 hearing-impaired individuals have been implanted with the Nucleus CI device worldwide; fewer than $1 \%$ of these have been implanted bilaterally. The vast majority of this group of bilateral cochlear implant users, being adults, were implanted in the second ear at the request and discretion of the patient and surgeon outside the realm of research in the pursuit of further improved hearing ability in the everyday situation.

Generally speaking, a better signal-to-noise ratio is needed by hearing-impaired subjects relative to that required by normal hearing subjects for the same level of speech comprehension. In addition, a relatively lower binaural advantage is noted for the hearing-impaired listener compared with that noted for the normal hearing person in spatially separate speech in noise conditions $(1,2)$. Nonetheless, bilateral implants are not actively promoted by most clinicians, possibly because of a combination of the additional costs involved coupled with the relative lack of published evidence to support the advantages over unilateral CIs. To investigate the potential clinical application of bilateral electrical stimulation for hearing-impaired adults on a broader scale, a multicenter prospective collaborative study was undertaken across German-speaking clinics in Germany and Switzerland. Subjects were enrolled after receipt of bilateral Nucleus implants in either simultaneous or sequential implant procedures. The effect of bilateral electrical stimulation on performance measures was evaluated and compared with performance with monaural electrical stimulation. The subsequent advantages of head-shadow effect and binaural signal processing were quantified. The study data presented in this article reflect the results of speech comprehension measures in quiet and in noise and the localization of sound.

\section{PATIENTS AND METHODS}

\section{Selection criteria}

Subjects enrolled were all 18 years of age or older, German native speakers who were postlinguistically deafened, and displayed a bilateral severe to profound sensorineural hearing loss. All subjects derived either no or very minimal benefit from conventional acoustic amplification either bilaterally or contralaterally to a previously implanted ear. Before bilateral implantation, either simultaneously or sequentially, all subjects were informed of the implications of being involved in the study evaluations and required to sign an informed consent form.

\section{Subjects}

Thirty-seven adults were enrolled in the study: 22 were implanted simultaneously and 15 sequentially. The sequentially implanted subjects had an average interimplant interval of 2.2 years $(\sigma \pm 1.4 \mathrm{yr}$; range, $0.4-5.6 \mathrm{yr})$. Of those sequentially implanted, 10 were implanted initially in the right ear and 5 in the left ear. The majority of subjects were progressively deafened, with a mean duration of deafness of 10.7 years $(\sigma \pm 14.1$ yr; range, $0.8-45 \mathrm{yr})$ for the right ear and 10.0 years $(\sigma \pm 13.2$ yr; rrange, $0.8-41 \mathrm{yr}$ ) for the left ear. Mean age at bilateral implantation for all subjects was 46 years $(\sigma \pm 14 \mathrm{yr}$; rrange, 18-67 yr). All subjects received the Nucleus 24 CI (Cochlear Ltd., Sydney, Australia) and used the Nucleus SPrint or ESPrit $3 \mathrm{G}$ speech processor. The majority of the subjects used the ACE speech coding strategy, with the remaining two using the SPEAK speech coding strategy.

\section{Study design}

Repeated single subject measures were carried out for each subject, with each subject serving as their own control. Speech perception tests were performed for unilateral stimulation of each ear and for bilateral stimulation in quiet and in three specific listening conditions in noise. Subjects were evaluated at three test intervals after switch on of the second implant at 1 month, 3 months, and 6 months.

For all tests performed unilaterally, the patient's speech processor volume was set on their preferred volume for each ear. As a consequence of the loudness summation effect when using both CI devices, individual processor maps were created for monaural and binaural listening conditions and subsequently adjusted to achieve a percept of balanced comfortable overall loudness for all maps. As a result, for test situations where the test stimulus/stimuli are presented at 0 degrees azimuth, thus arriving equally to each ear, the presence and subsequent influence of loudness summation, normally occurring in the binaural listening condition, was either removed or reduced to a minimum. Subsequently, any improvement noted in performance in the binaural listening condition compared with that for either monaural listening condition, termed "binaural advantage," was interpreted to be the result of binaural redundancy.

As the majority of CI users present with some inherent degree of asymmetry in their interaural hearing loss configuration or for the baseline speech comprehension ability, a better and a poorer performing ear to some degree is generally noted. Thus, the binaural advantage provided by bilateral stimulation for speech comprehension was calculated with respect to each ear separately, classified as a better or poorer performing ear for each material and in each test condition. The statistical significance of the interaural performance difference was cal- 
culated and taken into account for the interpretation of the binaural advantage.

For tests of localization, for which the directionality of the response is recorded and compared with the source of the stimuli in degrees, the binaural advantage was assessed by comparing the responses when bilaterally aided to those obtained for the unilateral fitting of each ear separately (i.e., versus right and left ears). It was not justified to use a better and poorer ear classification for the analysis of the localization test data, as there was no significant interaural difference noted for RMS error in degrees for the group. Statistical comparison of the data sets from each listening condition was performed for matched pairs using the Student's $t$ test.

\section{Test setup for speech comprehension}

Speech comprehension tests were performed via loudspeaker in a sound-treated room. Audiometers calibrated according to ISO 9000 were interfaced with a computer for presentation of the test stimuli via the loudspeakers. Sound-field tests were performed using two laterally positioned loudspeakers, 90 degrees apart, each at an angle of 45 degrees to either side of the subject's head when seated between the speakers at a distance of $1 \mathrm{~m}$. For speech perception tests in quiet, the speech stimuli were presented from one of the speakers with the subject facing the speaker in use at 0 degrees azimuth. Regardless of the speaker setup in use, the subject was instructed to maintain a relatively stable head position throughout the testing.

For testing in background noise, three alternate presentation setups were used: $\mathrm{S} 0^{\circ} \mathrm{N} 0^{\circ}, \mathrm{S}+45^{\circ} \mathrm{N}-45^{\circ}$, and $\mathrm{S}-45^{\circ} \mathrm{N}+45^{\circ}$. For $\mathrm{S}^{\circ} \mathrm{N}^{\circ}$, both the speech signal and competing noise signal were presented from the same speaker positioned at 0 degrees azimuth to the subject's head. For the remaining speech in noise conditions, the speech and noise signal were presented simultaneously from the spatially separated speakers at 90degree intervals, with the subject seated between the speakers. This test condition was described by Cox et al. (3) in 1981 as being most sensitive to the measurement of the binaural squelch effect, whereas a two-speaker setup presenting the speech stimuli directly in front of the subject at 0 degrees azimuth and the noise source lateral to the head at either +90 degrees is most effective in demonstrating the head-shadow effect. As the binaural squelch effect is considerably smaller than the head-shadow effect in absolute terms, the speaker setup most sensitive to the binaural squelch effect was selected for the study.

In the $\mathrm{S}+45^{\circ} \mathrm{N}-45^{\circ}$ condition, the speech signal is presented from the speaker to the right at 45 degrees azimuth, and the noise signal from the speaker to the left at -45 degrees azimuth to the subject's head. In contrast, for the $\mathrm{S}-45^{\circ} \mathrm{N}+45^{\circ}$ condition, the speech signal is presented from the speaker to the left and the noise signal from the speaker to the right of the subject's head. Alternating the presentation of spatially separated speech in noise permits both monaurally aided ears to be tested in both the relative superior and inferior signal-to-noise ratios.

\section{Speech materials}

Speech comprehension measures were performed using both open-set word lists in quiet and a choice of two open-set sentence materials in quiet and in noise. The choice of sentence materials mainly reflects the merger of two protocol designs, clinic preference, and German language dialect issues. In some cases, clinicians elected to test subjects with both types of sentence materials. All test materials were presented for bilateral and unilateral listening conditions for speech in noise tests at 3 and 6 months after bilateral implant. Testing in quiet was additionally requested at 1 month after switch-on.

Test materials were recorded without repetition on compact disk, with an additional track of the standard competing background noise made available with each type of sentence material. Specifically for the Hochmair-Schulz-Moser (HSM) sentences, the background noise that conforms to the standards of the Comite Consultatif International Telephonique (CCITT rec. 227), is an unmodulated speech-shaped noise with the same spectral envelope as the long-term average spectrum of speech (15). For the Oldenburger (OLSA) sentences, the competing background noise signal is a speech-simulating continuous noise that exactly matches the long-term spectrum of the sentence material, generated from 30 statistically controlled overlaps of the test words used $(16,17)$. Randomization of test lists presented for each patient across listening conditions and test intervals was carried out independently at each test site. The Freiburger monosyllabic word test, presented in quiet only at $70 \mathrm{~dB}$ sound pressure level (SPL), comprised two lists of 20 words for each listening condition, for which a percentage word correct score was obtained $(\mathrm{n} / 40 * 100)$. The OLSA sentences in quiet, also presented at $70 \mathrm{~dB}$ SPL, comprised two lists of 10 sentences, with each list consisting of 50 words. A percentage score correct was obtained for each listening condition $(\mathrm{n} / 100 * 100)$. For testing OLSA sentences in noise, an adaptive signal-to-noise test procedure was used presenting three lists of sentences with the competing background noise set at $65 \mathrm{~dB}$ SPL, and the speech level of each sentence was adaptively altered pending the individual's response to each test item, to obtain the signal-to-noise ratio (SNR) at which a $50 \%$ correct speech comprehension score is achieved. The resulting score for each listening condition is a critical SNR (i.e., $\mathrm{SNR}_{50 \%}$ ). Alternatively or additionally to the use of the OLSA sentences, testing in quiet and in noise was performed at some institutions using the HSM sentence lists. In quiet, the HSM sentences were presented at $70 \mathrm{~dB}$ SPL using one list of sentences for each of the three listening conditions and the percentage correct score recorded (n/106*100). In noise, one list of HSM sentences was presented against a fixed SNR of +10 , with the competing background noise set at $60 \mathrm{~dB}$ SPL. Similarly for tests in quiet, the HSM sentences in noise were scored for percentage correct word scores in each listening condition $(\mathrm{n} / 106 * 100)$.

\section{Tests of localization}

\section{Setup}

Tests of localization performed in an anechoic sound chamber with multiple speakers were carried out at one of three reference test centers with the appropriate facilities. Twelve equidistant speakers at intervals of 30 degrees, each identified with a visible number from 1 to 12 in a clockwise sequence, were located in a circle of $2 \mathrm{~m}$ diameter around the room in a horizontal plane approximately $1 \mathrm{~m}$ above the floor. For testing, the patient was seated in the center of the circle facing the speaker with number 12. For each listening condition, monaural right $\mathrm{CI}$, monaural left $\mathrm{CI}$, and binaural CIs, the test stimulus, a shortened sentence from the HSM sentence test, was presented from each speaker location a total of five times in a random sequence around the room. The three listening conditions were assessed at an intensity level between 65 and $70 \mathrm{~dB}$ SPL and/or between 55 and $60 \mathrm{~dB}$ SPL. The stimulus level selected ultimately depended on the subject's aided threshold for speech reception. Results from the preferred intensity level for each subject were combined for the calculation of the group's performance regardless of the intensity level used. For 
each response, the loudspeaker identified by the subject as delivering the sound was recorded, resulting in a total of 60 speaker location responses for each patient. The error for each response was subsequently converted to degrees and the RMS error for each subject and finally for the group in each listening condition.

\section{RESULTS}

Freiburger monosyllabic words in quiet

Matched pair data for all three listening conditions in quiet (i.e., unilateral CI in each ear and bilateral CIs) are available for the Freiburger monosyllabic word (FMW) test at each test interval, 1, 3, and 6 months after bilateral implant, for 30 subjects as shown in Figure 1. The poorer ear and the better ear were classified on the basis of the subject's performance on the FMW test at 1 month postoperatively and retained over the subsequent test intervals for the analysis. Comparing the results obtained for the 1-month and 6-month test intervals, there is significant improvement over time for all three listening conditions, that is, the poorer ear, the better ear, and bilateral listening conditions $(p<0.0005, p<0.005$, and $p<$ 0.006 , respectively). A significant binaural advantage is noted over the poorer ear of $16 \%, 17 \%$, and $9 \%$ at 1,3 , and 6 months, respectively, which is considered statistically significant $(p<0.00001$ at 1 and 3 months, $p=$ 0.00009 at 6 months). In comparison with the better performing ear, a mean binaural advantage is noted of $1 \%$, $6 \%$, and $0 \%$ at 1,3 , and 6 months, respectively, which is not considered clinically significant but is statistically significant at the 3-month test interval. In addition, at each test interval, a significant interaural score difference is observed, with the mean better ear scores being significantly superior to the mean poorer ear scores $(p=$ $0.00002, p=0.0001$, and $p=0.0003$ for 1,3 , and 6 months, respectively).

\section{OLSA sentences in quiet}

A comparison of test results from the test intervals at 3 and 6 months after the second implant switch-on was

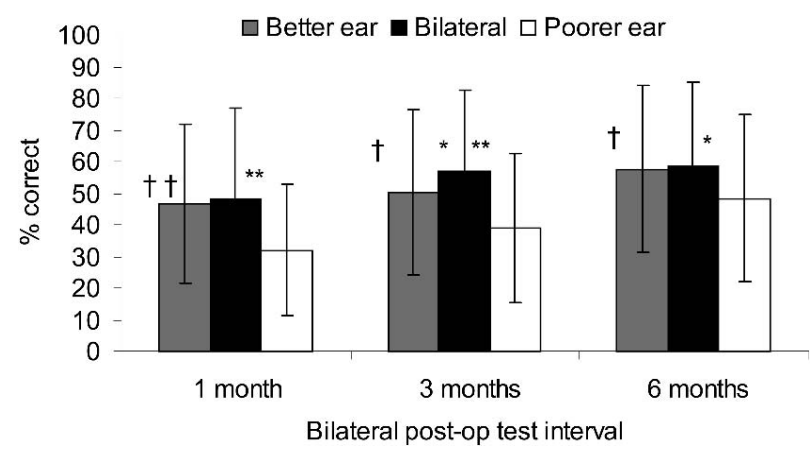

FIG. 1. Freiburger monosyllabic words in quiet. Mean correct percent scores at 1,3 , and 6 months after bilateral implant in each monaural and binaural listening condition $(\mathrm{n}=30)$. ${ }^{*} p$ 0.008 to 0.0003 , bilateral versus monaural; ${ }^{* *} p<0.00005$, bilateral versus monaural; $\dagger p \sim 0.0001$ to 0.0003 , significant interaural difference; $\uparrow \uparrow p<0.00005$, significant interaural difference.

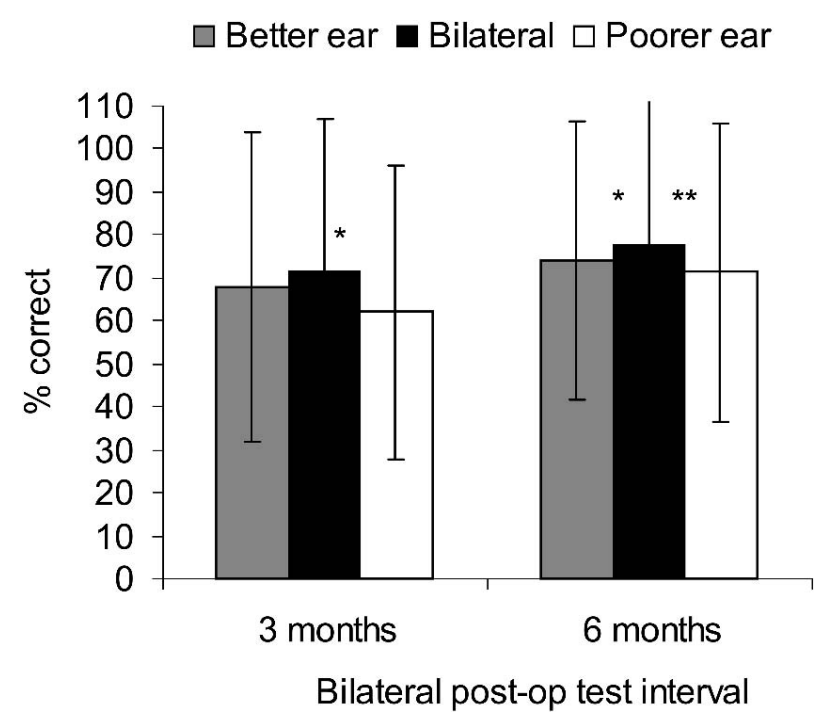

FIG. 2. Oldenburger sentence scores in quiet. Mean correct percent scores at 3 and 6 months after bilateral implant in each monaural and binaural listening condition $(n=19)$. ${ }^{\star} p \sim 0.004$ to 0.03 , bilateral versus monaural; ${ }^{\star \star} p=0.0001$, bilateral versus monaural.

available for data sets from 19 subjects with matched pair data for all three listening conditions for the OLSA sentences in quiet, as shown in Figure 2. Poorer and better performing ears were classified on the basis of performance at the 3-month test interval on the OLSA sentences in quiet and retained for the analysis of 6-month data also. Between 53 and $63 \%$ of the monaural listening scores for the better ear and $35 \%$ of those for the poorer ear were $80 \%$ correct or greater and thus present a ceiling effect on which it is difficult to measure improvement in the bilateral condition. Statistically, the data suggest a significant binaural redundancy effect at 3 months when comparing the mean score for bilateral stimulation to that for the poorer ear, with a gain of $9 \%(p=0.004)$, and in comparison with mean scores for the poorer ear and the better ear at 6 months, with a gain of $6 \%(p=0.0001)$ and $4 \%(p=0.03)$, respectively. At 3 months, a statistically significant interaural difference was observed, with the better ear scores being on average $6 \%$ greater than for the poorer ear $(p=$ $0.007)$. At 6 months, the interaural difference is no longer present. Over time, from 3 to 6 months after second implant switch-on, the subject group demonstrates a significant mean improvement in performance of $6 \%$ for the binaural and better ear listening conditions $(p=0.02$ and $p=0.04)$ and $9 \%$ for the poorer ear listening conditions $(p=002)$.

\section{OLSA sentences in noise}

Data for performance on the OLSA sentences in the three speech in noise test conditions is illustrated for the 6-month after second implant switch-on test interval for 20 subjects in Figure 3. Classification of better and poorer ears was made on the basis of the 3-month performance for each ear in the $\mathrm{S}^{\circ}{ }^{\circ} 0^{\circ}$ test condition and 


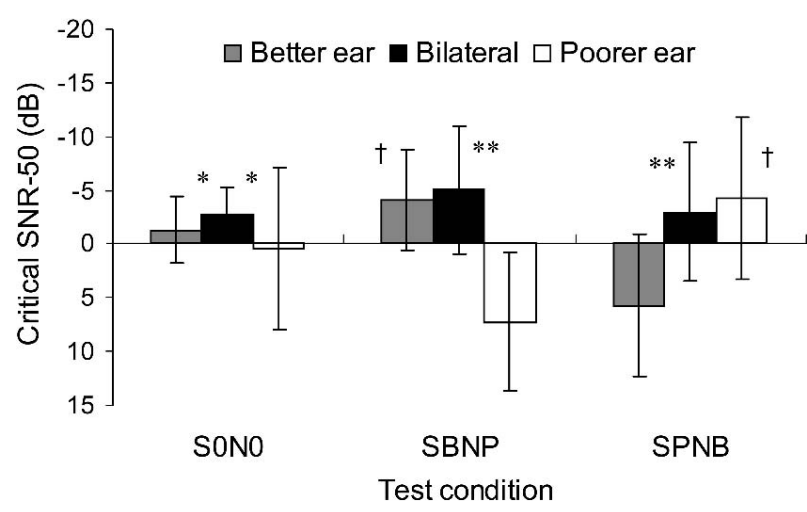

FIG. 3. Oldenburger sentences in adaptive SNRs. Mean performance scores at 6 months for bilateral and unilateral listening conditions $(n=20)$. Conditions: SONO, speech and noise 0 degrees azimuth; SBNP, speech at better ear, noise at poorer ear; SPNB, speech at poorer ear, noise at better ear. ${ }^{*} p \sim 0.01$ to 0.04 , bilateral versus monaural; ${ }^{* *} p<0.00005$, bilateral versus monaural; $\uparrow p<0.00005$, significant interaural difference.

retained for analysis of performance for each of the spatially separated speech in noise test conditions at the 3and 6-month test intervals. For the subjects for whom no 3-month performance measure was carried out, the 6-month performance data were used to classify each ear. The graphically presented data represent the mean and standard deviation of the critical SNR required for $50 \%$ correct speech understanding scores for the group for each listening condition in each of the three test conditions. A negative $\mathrm{SNR}_{50 \%}$ (presented graphically by bars above the $x$ axis) suggests that, on average, a $50 \%$ correct score was achievable with the speech level presented softer than the noise signal level (i.e., a more difficult $\mathrm{SNR}$ ). A positive $\mathrm{SNR}_{50 \%}$ (presented graphically by bars below the $x$ axis) suggests that, on average, a 50\% correct score was achievable with the speech level presented louder than the noise signal level (i.e., an easier SNR). As the data suggest, when binaurally stimulated, 50\% speech comprehension is achievable with a more difficult SNR compared with when monaurally stimulated. In the $\mathrm{S}^{\circ}{ }^{\circ} 0^{\circ}$ condition, with each ear being presented with the same SNR, interaural performance scores for the unilateral listening conditions are not significantly different $(p=0.3)$. The effect of binaural redundancy is statistically significant over the mean better ear $\mathrm{SNR}_{50 \%}$ (mean, $-1.4 \mathrm{~dB}, \sigma=2.3 \mathrm{~dB}$; range, $-7.1-0.8 \mathrm{~dB} ; p=0.01)$ and over the mean poorer ear $\mathrm{SNR}_{50 \%}$ (mean, $-3.2 \mathrm{~dB}, \sigma=$ $6.2 \mathrm{~dB}$; range, $-22.8-1.8 \mathrm{~dB} ; p=0.04)$.

In spatially separate speech in noise conditions, the binaural advantage is significant over monaural listening conditions when presented with the inferior SNR (i.e., when the test ear is closest to the noise source) ( $p<$ $0.00005)$. It is assumed that this advantage noted for the binaural listening condition is largely due to the headshadow effect resulting from the physical properties of the head attenuating the noise on the contralateral side, creating an even more favorable SNR for the ear closest to the speech signal, which ultimately becomes the ear the subject attends to in such a situation. The resulting bilateral head-shadow benefit was calculated by comparing the $\mathrm{SNR}_{50 \%}$ for each ear in each spatially separate speech in noise test condition (i.e., $\mathrm{SNR}_{50 \%}$ for the ipsilateral ear closest to the speech source minus $\mathrm{SNR}_{50 \%}$ for the contralateral ear closest to the noise source). This resulted in a mean bilateral head-shadow benefit of -11.4 $\mathrm{dB}(\sigma=6 \mathrm{~dB} ; p<0.00001)$ when the better ear is closest to the speech source and a mean benefit of -10 $\mathrm{dB}(\sigma=8 \mathrm{~dB} ; p<0.00001)$ when the poorer ear is closest to the speech source. Comparison of the $\mathrm{SNR}_{50 \%}$ for bilateral stimulation versus the $\mathrm{SNR}_{50 \%}$ for the unilateral ear when closest to the speech source resulted in a mean improvement of $-0.9 \mathrm{~dB}(\sigma=4.1 \mathrm{~dB}$; range, $-15.1-7.6 \mathrm{~dB}$ ) relative to the better ear and a mean decrement of $1.3 \mathrm{~dB}(\sigma=6.0 \mathrm{~dB}$; range, $-11.5-16.5 \mathrm{~dB})$ relative to the poorer ear, neither of which was considered statistically significant $(p=0.3)$. This difference is also below the 2- $\mathrm{dB}$ minimal difference suggested by the developers of the OLSA sentence materials to overcome the inherent test-retest variability $(16,17)$. Thus, no significant binaural squelch effect was observed for the group.

\section{HSM sentences in quiet}

Matched pair data sets were available for a comparison of test results from the test intervals at 3 and 6 months after second implant switch-on for 14 subjects as shown in Figure 4. A binaural advantage is noted over the poorer ear at 3 and 6 months of $15 \%(\sigma=19 \%)$ and $13 \%(\sigma=16 \%)$, respectively, which is considered statistically significant $(p=0.01)$. In comparison with the better performing ear, a mean improvement for binaural scores is noted of $2.9 \%$ at 3 months and $1.6 \%$ at 6 months, neither of which is considered statistically significant $(p>0.3)$. Subsequently, there is no measurable binaural redundancy effect noted. As for the individual scores for OLSA sentences, a proportion of the speech

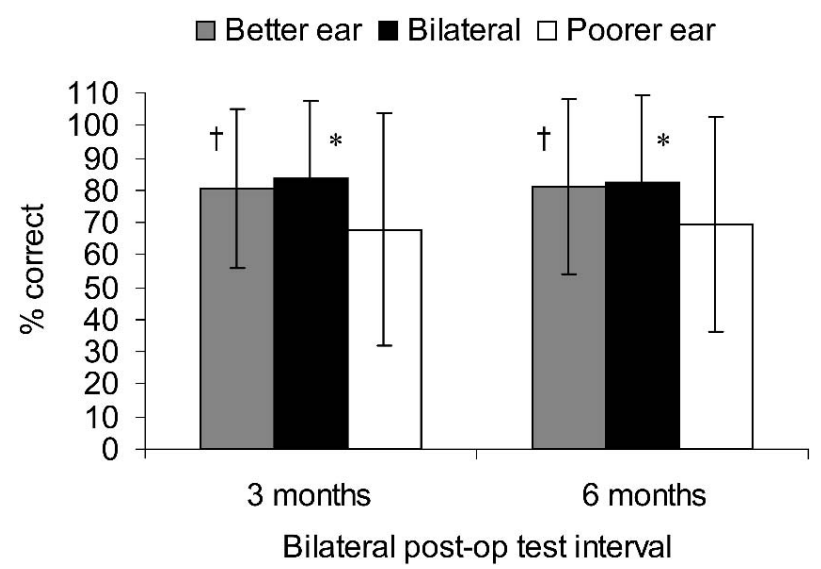

FIG. 4. HSM sentence percent correct scores in quiet. Mean correct percent scores at 3 and 6 months after bilateral implant in each monaural and binaural listening condition $(\mathrm{n}=14)$. ${ }^{*} p$ 0.01 , bilateral versus poorer ear; $\uparrow p<0.04$, significant interaural difference. 
comprehension scores for the monaural listening conditions for the HSM sentences present a potential ceiling effect, with $64 \%$ of the scores for the better ear and $57 \%$ of those for the poorer ear being greater than $80 \%$ correct and thus making it difficult to measure a binaural advantage. A significant interaural difference is noted at both test intervals, with superior performance noted for the better ear $(p=0.03$ and $p=0.04$ at 3 and 6 months, respectively). In view of the interaural differences observed for mean monaural performance scores, the significant binaural advantage relative to the poorer ear is potentially a consequence of the subjects attending to the better ear. No significant change in performance was noted over time for any of the listening conditions.

\section{HSM sentences in noise}

Data for performance on the HSM sentences in the three speech in noise test conditions for the 6 months post-bilateral switch-on test interval is shown for 23 subjects in Figure 5. Classification of better and poorer ears was made on the basis of the 3-month performance for each ear in the $\mathrm{S}^{\circ} \mathrm{N}^{\circ}$ test condition and retained for analysis of performance for each of the spatially speech in noise test conditions throughout. For the subjects for whom no 3-month performance measure was carried out, the 6-month performance data were used to classify each ear. The graphically presented data represent the mean scores and 1 standard deviation for each of the listening conditions in each of the three test conditions for speech in noise at a fixed $\mathrm{SNR}$ of +10 . In the $\mathrm{S}^{\circ} \mathrm{N}^{\circ}$ condition, although the effect of binaural redundancy on comprehension scores when bilaterally stimulated is not significant relative to the comprehension scores achieved for stimulation of the better ear alone, showing a mean difference of $4 \%(\sigma=11.8 \%$; range, $-15-35 \% ; p=0.1)$, it is considered significant relative to scores achieved for stimulation of the poorer ear alone, with a mean difference of $26 \%$ ( $\sigma=18 \%$; range, $0-67 \% ; p<0.00005)$. As

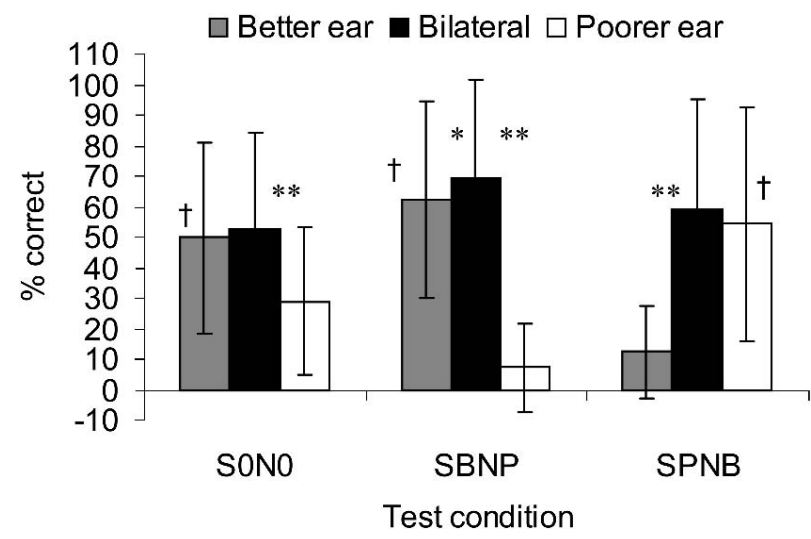

FIG. 5. HSM sentence percent correct scores in noise at 6 months. Mean performance scores for bilateral and unilateral listening conditions $(n=23)$. Conditions: SONO, speech and noise 0 degrees azimuth; SBNP, speech at better ear, noise at poorer ear; SPNB, speech at poorer ear, noise at better ear. ${ }^{*} p \sim 0.02$, bilateral versus monaural; ${ }^{* *} p<0.0005$, bilateral versus monaural; $\uparrow p<0.0005$, significant interaural difference. there is significant interaural difference between the scores in the $\mathrm{S}^{\circ} \mathrm{N} 0^{\circ}$ condition $(p=0.0001)$, it is possible that the binaural benefit over the poorer ear is a result not of true binaural redundancy but of the subject attending to the better ear.

In spatially separate speech in noise conditions, a significant improvement in speech comprehension scores when bilaterally stimulated is observed over scores when monaurally stimulated and the test ear is closest to the noise source (i.e., a binaural advantage is noted over each ear alone when presented with the inferior SNR $(p<$ 0.00005). Comparison of speech scores achieved for bilateral stimulation versus those for the unilateral stimulation of the ear closest to the speech source resulted in a significant mean improvement of $8 \%(\sigma=13 \%$; range, $-11-35 \%)$ relative to the better ear $(p=0.02)$ and a mean improvement of $4 \%(\sigma=12 \%$; range, $-15-41 \%)$ relative to the poorer ear $(p=0.1)$, The significance of the binaural advantage for speech comprehension over the scores for the better ear alone despite the superior SNR suggests contribution of the auditory information received from the poorer ear with an inferior SNR. That is, a significant binaural squelch effect is observed over the better ear when closest to the speech signal.

Whether the better or poorer performing ear is provided with the superior SNR, a significant interaural performance difference arises that favors speech comprehension in the ear with the superior SNR $(p<0.00005)$, suggesting a significant bilateral head-shadow benefit. Specifically, a mean bilateral head-shadow benefit of $55 \%(\sigma=29 \%$; range, $0-96 \%)$ for the better ear when closest to the speech source and a mean of $42 \%(\sigma=$ $36 \%$; range, $-22-98 \%$ ) for the poorer ear when closest to the speech source.
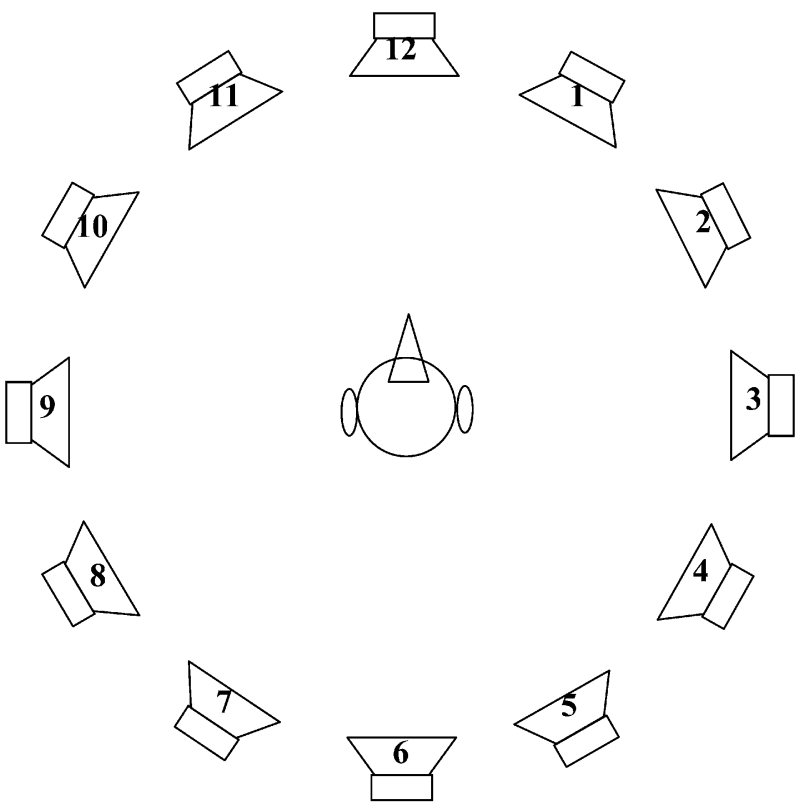

FIG. 6. Speaker setup for tests of sound localization. 


\section{Localization results}

Localization data are available for a total of 16 patients. Figure 6 illustrates the speaker locations for visual clarification of the test setup. The response data to the lower stimuli intensity was used for the group analysis (i.e., 55-60 dB SPL) for the majority of subjects, with two exceptions (i.e., Subjects S13, S15, for whom $65 \mathrm{~dB}$ SPL was preferred in order to make the stimuli comfortably audible for the localization task for each ear). Figure 7 demonstrates the mean error, RMS to the left and right for each speaker location from 1 to 12 corresponding to 30 degrees to $360 / 0$ degrees, respectively, in a clockwise direction. The diagonal line represents the ideal response when equal to the stimuli location for each speaker. The mean group responses for the binaural listening condition provide a better approximation of the stimuli location relative to either monaural condition. This was the case for 15 of the 16 subjects. Generally speaking, when monaurally aided in the left ear, response error mostly occurs toward the left side for stimuli presented on the right side of the subject's head; when monaurally aided in the right ear, response error mostly occurs toward the right side for stimuli presented on the left side of the subjects head. The RMS, which takes into account the degree of error in the response to each stimulus location was, on average, 87 degrees for the left monaural CI condition (range, $70-102$ degrees; $\sigma=8.9$ degrees), 89 degrees for the right monaural condition (range, 64-103 degrees; $\sigma=9.8$ degrees), and 50 degrees for the binaural condition (range, 16.0-99 degrees; $\sigma=23.9$ degrees). This results in a ratio for the calculated RMS for bilateral and monaural listening conditions of 1:1.8. Examination of individual results revealed that binaural localization ability was superior to monaural localization ability for all but one subject (S19). Statistically, the responses between the monaural conditions are not significantly different $(p=0.3)$ for the group, whereas binaural localization responses are significantly superior compared with those for either monaural condition $(p<$

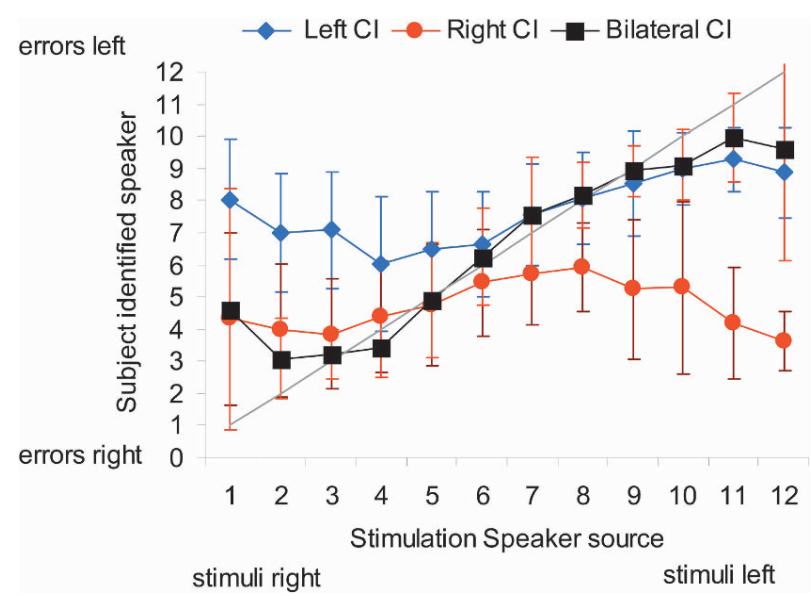

FIG. 7. Localization in the sound field. Mean responses for sound localization in unilateral and bilateral listening conditions $(n=16)$.
0.00005). On further examination of individual results for monaural and binaural listening conditions, the RMS was at least 60 degrees for all subjects when monaurally stimulated. For bilateral stimulation, the RMS was below 30 for 3 of 16 subjects and below 60 degrees for 14 of 16 subjects.

\section{DISCUSSION}

The head-shadow effect was demonstrated as a relatively robust and significant bilateral benefit for the vast majority of the group for the comprehension of both the OLSA and HSM sentences in spatially separate speech in noise test conditions. The bilateral head-shadow benefit, although not a binaural processing effect, is an important advantage of bilateral stimulation, as it permits the CI user the flexibility to attend to the ear with the superior SNR in the real listening environment. This is especially important when presented with speech in noise in an environment that cannot be easily altered to suit the monaurally aided listener such as in a car, in a theatre, at a dinner table, at a party, at a meeting, or walking alongside a busy street.

The two effects that suggest true binaural processing of auditory information arriving at each ear are binaural redundancy and binaural squelch. Binaural squelch, demonstrable only in the spatially separate speech in noise test condition, was not as robust or consistently measured for either type of sentence materials used as was the bilateral head-shadow benefit, which agrees with the findings from other studies $(10,12)$.

A significant binaural squelch effect was not demonstrated for the group for OLSA sentences in noise, whereas $15 \%$ of the individuals tested demonstrated a greater than 1- to 2-dB difference in the $\mathrm{SNR}_{50 \%}$ for bilateral and unilateral listening conditions. Taking into account that the required difference in $\mathrm{SNR}_{50 \%}$ between test conditions is reduced to below $1 \mathrm{~dB}$ as a result of the two training lists performed before each data collection, the proportion of subjects demonstrating a significant squelch effect is increased to $38 \%(16,17)$. However, the significance of such small differences is difficult to establish statistically for a group of only 20 subjects and subsequently suggests that much larger sample sizes of subjects may be required in future studies.

For the HSM sentences at SNR +10 , a binaural squelch effect was noted for $38 \%$ of the individual subject data comparing the binaural listening condition results to the better ear scores when presented with the superior SNR, resulting in a barely significant binaural squelch effect for the group $(p=0.02)$. In view of the high proportion of subjects with ceiling and floor effects as described previously, testing the same group of subjects with the HSM sentences in noise via an adaptive procedure to determine the critical $\mathrm{SNR}_{50 \%}$ for speech comprehension could potentially result in improved statistical significance of the binaural squelch effect for the group over both ears. In addition, larger subject sample sizes would increase the power of the significance of 
the performance differences between the different listening conditions.

Although loudness balancing was carried out for all subjects' processors, it is not possible to guarantee the absence of any loudness summation of the signals from both processors when used simultaneously that may potentially have assisted in improving speech comprehension of speech in noise presented coincidentally compared with that observed when unilaterally stimulated. Assuming that loudness balancing was achieved, the improvement for speech comprehension scores for the bilateral listening condition over the monaural listening conditions when each ear received potentially the same signal as in quiet at 0 azimuth or at $\mathrm{S}^{\circ} \mathrm{N}^{\circ}$ could be referred to as a binaural advantage. Whether the benefit is due to loudness summation or binaural redundancy, it is only made available through the use of two devices. In our study, this resulted in a significant improvement in binaural scores for the OLSA sentences in quiet and an improved SNR for the $\mathrm{SNR}_{50 \%}$ over the scores for the poorer ear at 3 and 6 months. In comparison with the better ear, a binaural advantage was also observed for scores in quiet and in noise for the $\mathrm{SNR}_{50 \%}$ at the 6-month test interval only, at which time the interaural difference between the individual ear scores was no longer significant.

Although noncompliance with the protocol excluded the possibility of including performance in noise data for several subjects, for four subjects (S6, S9, S13, and S29), all considered poor performers $(<35 \%$ correct for FMW in quiet monaurally and binaurally), it was not possible to perform testing in noise, as they were not able to achieve a minimum score of $50 \%$ correct required by the adaptive procedure for testing OLSA sentences in noise used at the implant sites. On closer examination, all four subjects had undergone simultaneous procedures and all had a duration of deafness between 28 and 40 years, which is considerably longer than the mean duration for the group, which is between 10 and 10.7 years. Nonetheless, it is not possible to draw a correlation between the duration of deafness alone and the performance of subjects at 6 months after second implant, as several other subjects with the study group with equally long duration of deafness were able to be tested in noise to determine the $\mathrm{SNR}_{50 \%}$ at both 3- and 6-month test intervals and were subsequently not considered to be poor performers.

After closure of the study, additional performance data were available for Subject S29 at 12 months after surgery for the FMW lists in quiet and for the OLSA sentences in quiet and in noise. The data suggest stable performance from 6 to 12 months with respect to scores on the FMW lists in quiet, remaining below 25\%; however, for the OLSA sentences in quiet, a marked improvement over time was observed, with scores originally being below $16 \%$ at 6 months, and subsequently increasing to $67 \%$ for the binaural condition and 44 to $47 \%$ for the monaural conditions. Testing comprehension in noise for the OLSA sentences at $\mathrm{SNR}_{-50 \%}$, previously not possible, also suggested further development of the binaural listening advantage, with a binaural advantage over monaural listening conditions for the SNR for the measured $\mathrm{SNR}_{50 \%}$ of 7 to $8 \mathrm{~dB}$ with the speech signal closest to the right ear and 1 to $2 \mathrm{~dB}$ with the speech signal closest to the left ear. This implies the need to extend the evaluation interval of performance when trying to determine the binaural advantage for future studies, perhaps for all subjects, but particularly for poorer performers with simultaneous bilateral implant procedures and prolonged duration of deafness (e.g., $>20 \mathrm{yr}$ ).

A post hoc analysis of the relative effect of the sequence of bilateral implantations on performance was not conclusive in view of considerably small subgroup sizes in either simultaneous or sequential subgroups. The trend across the test results for each subgroup suggests the same relative improvements in performance for the bilateral condition over unilateral conditions as noted for the larger study group. The difficulty experienced by some subjects both in quiet and in noise may also suggest that for cases with prolonged duration of deafness, the potential benefit of two implants may be better observed in sequential procedures rather than simultaneously.

Despite the existence of research into the benefits of bilateral stimulation for bilaterally implanted CI subjects to specifically measure the binaural advantages in spatially separate speech in noise conditions, it is very difficult and largely inappropriate to draw comparisons with studies using varying test setups, intensity levels, noise signals, recordings, mapping procedures for individual speech processors, speech coding strategies, and speech materials in the same or other languages.

A fraction of the data reported by other studies from German-speaking regions suggests use of the same German language FMW materials and the HSM sentence materials at the same intensity levels in quiet and in noise. Nonetheless, the speaker setup and test recordings may vary, as this is not always specified, and subsequently influence the outcomes $(8,9,14)$. Although these previously published studies consistently demonstrated a binaural advantage for speech comprehension of the HSM sentences at a fixed SNR of $+10 \mathrm{~dB}$, either as a result of large head-shadow effects and/or smaller binaural squelch effects, the absolute mean group scores for the monaural and binaural listening conditions in noise test conditions also varies.

From our subject group, mean performance scores for the HSM sentences at $+10 \mathrm{~dB}$ SPL at 6 months postoperatively with the speech signal closest to the better ear and noise signal closest to the poorer ear resulted in a mean better ear score of $62 \%(\sigma=32 \%)$ and a mean bilateral listening score of $70 \%(\sigma=33 \%)$, resulting in a statistically significant binaural advantage of $8 \%$. This same group of subjects demonstrated mean HSM sentences scores in quiet at $70 \mathrm{~dB}$ SPL of $84 \%(\sigma=24 \%)$ for the better ear and $85 \%(\sigma=23 \%)$ for the binaural listening condition.

The study by Stark et al. reports for 17 subjects implanted with the Med-El device, across three centers, 
results from HSM sentences at a $+10 \mathrm{~dB}$ SNR with an unspecified speaker setup, a mean better ear score of $23 \%(\sigma=16 \%)$ and a mean bilateral listening score of $42 \%(\sigma=17 \%)$, resulting in a statistically significant binaural advantage of $21 \%$. This same group demonstrates mean HSM sentence scores in quiet at $70 \mathrm{~dB}$ SPL of $80 \%(\sigma=18 \%)$ for the better ear and $86 \%(\sigma=$ $14 \%)$ for the binaural listening condition. The Schön et al. study reports on HSM scores at +10 SNR for nine subjects from a single site, also implanted with the MedEl device, using a four-speaker setup, and demonstrates results consistent with those by Stark et al., indicating a mean better ear score of $22 \%(\sigma=15 \%)$ and a mean bilateral score of $43 \%(\sigma=22 \%)$, resulting in a significant bilateral advantage of $21 \%$.

Clearly, all studies show a bilateral advantage for speech comprehension in noise for the same sentence materials despite the use of differing test methods for the different subject groups. Although there is good consistency between the absolute results and subsequent binaural advantage reported for comprehension of speech in noise between the Stark et al. and Schön et al. studies, the data from our study suggest higher mean scores for the better ear and bilateral conditions but a smaller bilateral advantage. This is despite the comparable mean performance in quiet for HSM sentences reported in this study and that reported by Stark et al.

This not only demonstrates the possible effects of differing test setups and test materials on performance in noise as mentioned previously, but also the potential influence of patient variables, hearing loss characteristics, group size, and speech coding strategy. Ideally, tests in noise should avoid the occurrence of performance ceiling and floor effects for any individuals, as they prevent effective measurement of the bilateral listening advantage. Subsequently, adaptive speech in noise testing performed at $\mathrm{SNR}_{50 \%}$ is preferable to testing at a fixed SNR.

The results from localization measures in this study demonstrate an improvement in the bilateral listening condition compared with either monaural listening condition for $94 \%$ of the subjects, resulting in a statistically significant mean improvement for the group of approximately 38 degrees in the RMS error in the binaural listening condition compared with the monaural listening conditions. The ratio of improvement of the RMS in the binaural and monaural listening conditions, at approximately $1: 1.8$, is slightly lower than the relative ratio of improvement noted for the binaural RMS over monaural RMS mean values from other published studies. Despite the possible influence of subject variability; differing speaker numbers, locations, and intervals; alternate test stimuli; and so forth, all studies consistently demonstrate a statistically significant advantage for localization when binaural stimulation is provided over monaural stimulation (10-12).

Only one subject within the subgroup tested did not demonstrate a binaural advantage for localization ability, Subject S19. This patient was reported to have encountered additional illnesses and confounding psychological factors that are assumed to have potentially affected the subject's attention span for the localization and speech comprehension tasks. In addition to the absence of binaural advantage for localization, Subject S19 was noted to display relative poor performance in all listening conditions for speech comprehension of both sentence and word materials, with no measurable differences between performance in monaural and binaural listening conditions observed.

\section{CONCLUSION}

The test results from this study provide evidence of binaural sound processing advantages provided via bilateral electrical stimulation over those provided via unilateral electrical stimulation in quiet and in noise, at least for a portion of the subjects for speech comprehension, and for improved localization ability for the majority of subjects tested. Over the 6-month period, a trend toward improved mean results via bilateral stimulation is observed, with significant improvement noted for group mean results for the poorer ear alone in most test conditions.

Although the head-shadow effect resulting in a bilateral head-shadow benefit appears to be the most robust and obvious advantage for the majority of subjects, binaural redundancy and binaural squelch, being smaller in ultimate effect, and demonstrated for some of the subjects, are not always demonstrated for the group and are more sensitive to the test methods used. Nonetheless, all of these benefits described, ultimately leading to improved speech understanding, are only possible through bilateral listening. Coupled with the added advantage of improved localization skills in the binaural listening condition, in the real world, the subject would be able to locate, turn toward, and attend to the desired signal more readily when binaurally stimulated, as well as combat the interfering noise signal with a combination of the physical properties of the head shadowing the ear with the superior SNR and additionally permitting binaural processing to occur pending the coincidence or spatial separation of the desired signal versus the interfering auditory signals.

In summary, bilateral electrical stimulation permits binaural auditory processing advantages that are not available through monaural listening, which may result in improved comprehension of speech and localization of sound as it occurs in the everyday listening situations, particularly amid background noise but also in quiet. In addition, being bilaterally implanted, the subject has greater listening flexibility, enabling him or her to take maximal advantage of the ear presented with the "superior" SNR as it occurs interchangeably within a nonstatic sound environment.

Acknowledgments: The authors thank the team members from the clinics involved in the study and all participating subjects for their cooperation 


\section{REFERENCES}

1. Festen JM, Plomp R. Speech reception threshold in noise with one and two hearing aids. J Acoust Soc Am 1986;79:465-71.

2. Dillon H. Hearing Aids. New York: Thieme, 2001:370-440.

3. Cox RM, De Chicchis R, Wark DJ. Demonstration of binaural advantage in audiometric test rooms. Ear Hear 1981;2:194-201.

4. Snik AF, Beynon AJ, Van der Pouw CT, Mylanus EA, Cremers $\mathrm{CW}$. Binaural application of the bone-anchored hearing aid. Ann Otol Rhinol Laryngol 1998;107:187-93.

5. Hawley ML, Litovsky RY, Colburn HS. Speech intelligibility and localization in a multi-source environment. J Acoust Soc Am 1999; 105:3436-48.

6. van Hoesel RJ, Clark G. Speech results with a bilateral multichannel cochlear implant subject for spatially separated signal and noise. Austral J Audiol 1999;21:23-8.

7. Gantz BJ, Tyler RS, Rubinstein JT, et al. Binaural cochlear implants placed during the same operation. Otol Neurotol 2002;23: 169-80.

8. Muller J, Schon F, Helms J. Speech understanding in quiet and noise in bilateral users of the MED-EL COMBI 40/40+ cochlear implant system. Ear Hear 2002;23:198-206.

9. Schon F, Muller J, Helms J. Speech reception thresholds obtained in a symmetrical four-loudspeaker arrangement from bilateral users of MED-EL cochlear implants. Otol Neurotol 2002;23:710-4.
10. Tyler RS, Gantz BJ, Rubinstein JT, et al. Three-month results with bilateral cochlear implants. Ear Hear 2002;23(1 Suppl):80-9.

11. Van Hoesel R, Ramsden R, O'Driscoll M. Sound-direction identification, interaural time delay discrimination, and speech intelligibility advantages in noise for a bilateral cochlear implant user Ear Hear 2002;23:137-49.

12. Van Hoesel RJ, Tyler RS. Speech perception, localization, and lateralization with bilateral cochlear implants. J Acoust Soc Am $2003 ; 113: 1617-30$

13. Van Hoesel RJ, Clark GM. Psychophysical studies with two binaural cochlear implant subjects. J Acoust Soc Am 1997;102:495507

14. Stark T, Muller J, Vischer M, et al. Multicenter study on bilateral implantation. In Cochlear Implant: An Update. The Hague, The Netherlands: Kugler, 2002:523-6.

15. Hochmair Desoyer I, Schulz E, Moser L, Schmidt M. The HSM sentence test as a tool for evaluating the speech understanding in noise of cochlear implant users. Am J Otol 1997;18(Suppl 6):83.

16. Wagener K, Brand T, Kollmeier B. Development and evaluation of a German sentence test: Part III. Evaluation of the Oldenburg sentence test. Z Audiol 1999;38:86-95.

17. Wagener K, Kuhnel K, Kollmeier B. Development and evaluation of a German sentence test: I. Design of the Oldenburger sentence test. Z Audiol 1999;38:4-15. 